1 Scale-up model obtained from the rheological analysis of highly concentrated

2 emulsions prepared at three scales

3 Anna May-Masnou*, Jordi Ribó-Besolí, Montserrat Porras, Alicia Maestro, Carme González,

4 José María Gutiérrez

5 Departament d'Enginyeria Química, Universitat de Barcelona, c/ Martí i Franquès, 1-11, E-

6 08028, Barcelona, Catalunya, Spain

$7 \quad$ *Corresponding author: e-mail: anna.may@ub.edu, Tel: +34 9340 39789; Fax: +34 934021291.

\title{
8 Abstract
}

9 We examine the scale invariants in the preparation of highly concentrated w/o

10 emulsions at different scales and in varying conditions. The emulsions are characterized

11 using rheological parameters, owing to their highly elastic behavior. We first construct and validate empirical models to describe the rheological properties. These models yield a reasonable prediction of experimental data. We then build an empirical scale-up model, to predict the preparation and composition conditions that have to be kept constant at each scale to prepare the same emulsion. For this purpose, three preparation scales with geometric similarity are used. The parameter $N \cdot D^{\alpha}$, as a function of the stirring rate $N$, the scale ( $D$, impeller diameter) and the exponent $\alpha$ (calculated empirically from the regression of all the experiments in the three scales), is defined as the scale invariant that needs to be optimized, once the dispersed phase of the emulsion,

20 the surfactant concentration, and the dispersed phase addition time are set. As far as we 21 know, no other study has obtained a scale invariant factor $N \cdot D^{\alpha}$ for the preparation of highly concentrated emulsions prepared at three different scales, which covers all three scales, different addition times and surfactant concentrations. The power law exponent 
obtained seems to indicate that the scale-up criterion for this system is the power input per unit volume $(P / V)$.

Keywords: Scale invariants; Scale-up model; Highly concentrated emulsions; Rheological parameters; Geometric similarity.

\section{Introduction}

Emulsions are prepared and used currently in many applications. They are used as templates for the synthesis of porous materials [1-6]. A specific, monodisperse and known pore size is usually desired. It is thus essential to monitor and predict the emulsion properties such as droplet size and size distribution, as they determine the pore or particle size and specific surface area of the product. Emulsion preparation is delicate, since a small variation in the procedure can change the final result. In this study, we work with highly concentrated water-in-oil (w/o) emulsions - used as a template for preparing hollow silica spheres - to determine how the process variables and, more importantly, the preparation scale, influence the final product. We performed a scale-up study using three scales with geometric similarity, and a scale ratio of 1:2:4. The largest tank we used has a capacity of $6 \mathrm{~L}$.

Emulsions are characterized, once the dispersed phase is set, by their droplet size and droplet size distribution. In a previous study [7], we examined the influence of the process variables on these parameters. We concluded that they were mainly influenced by the stirring rate and the surfactant concentration. We also studied the scale-up effect, but using only two different scales (1:2). Droplet size was measured by optical microscopy.

Another interesting feature of these emulsions is their rheological behavior. Many studies deal with the rheological properties of highly concentrated emulsions [8-14], also called gelemulsions [15-17], since they possess distinct flow behavior: they have a high yield stress $\tau_{0}$, are highly elastic $\left(G^{\prime}>>G^{\prime \prime}\right)$ and they have high viscosity $\eta$, which decreases with shear rate (shear-thinning). Regarding the large amplitude oscillatory shear (LAOS) behavior, these 
emulsions behave as type III fluids, or weak strain overshoot, in which only $G$ "' shows strain overshoot $[18,19]$, whereas $G^{\prime}$ is constant in the linear viscoelastic region (LVR) and then decays. The critical stress $\left(\tau_{c}\right)$ at which the loss modulus is maximum $\left(G{ }^{\prime}{ }_{\max }\right)$ in the oscillatory shear test is assimilated to a yield stress by Jager-Lézer et al. [10], since it confirms the transition from the elastic to the viscous region.

In [7], we obtained some empirical models that described the emulsion droplet size and polydispersity as a function of the process variables at two different scales. Here we derive empirical models and perform the scale-up study with the rheological parameters of the emulsions (viscosity, yield stress, viscoelastic parameters...), which are acquired using a rheometer.

The experimental design pursues the scale-up criteria for systems of this kind, particularly the scale-up invariant related to the stirring rate and the preparation scale.

\section{Experimental section}

\subsection{Materials}

Dodecane $(99.5 \%)$, which constitutes the emulsion continuous phase, and Span80®, which is the surfactant $(\mathrm{HLB}=4.3)$, were both purchased from Sigma-Aldrich. Deionized Milli-Q water constitutes the dispersed phase, which is $90 \%$ of the emulsion weight ( $88 \% \mathrm{vol} / \mathrm{vol})$.

\subsection{Preparation of highly concentrated emulsions}

Emulsions were prepared in three geometrically similar glass jacketed vessels (Fig. 1-a), agitated with a three-level P-4 pitched blade impeller (Fig. 1-b). The installation is the same as the one described in [7]. 

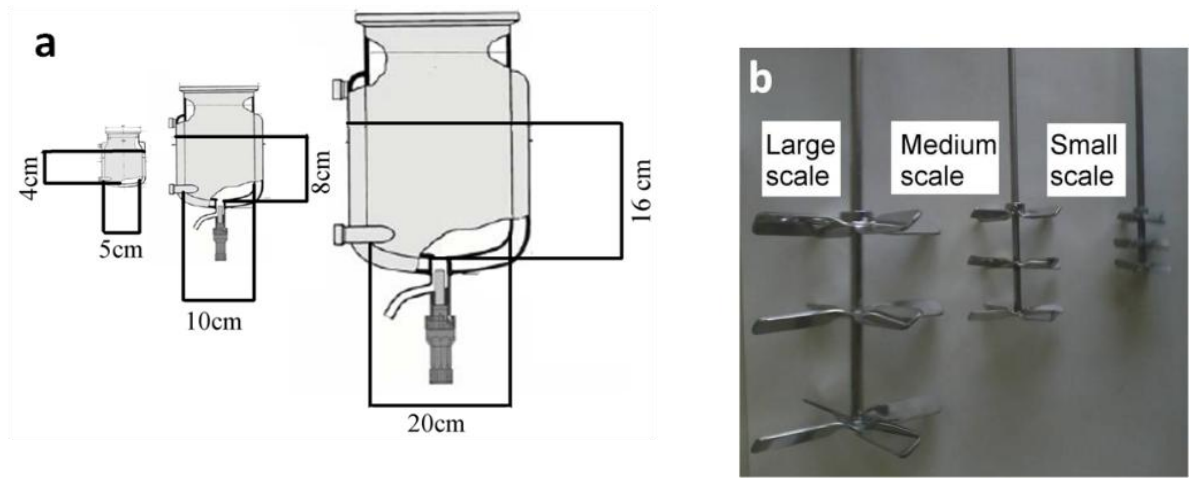

Fig. 1. (a) Liquid height and vessel diameter of the three scales. (b) Impellers used at the three scales.

The w/o emulsions were prepared following a two-step batch method: addition of dispersed phase and droplet breakup, followed by emulsion homogenization. The continuous phase is prepared beforehand by weighing, mixing and introducing into the vessel, which is already at the desired temperature, a certain amount of surfactant and oil. The impeller is placed slightly above the continuous phase in order to start the emulsification as soon as the dispersed phase is transferred to the vessel. While the dispersed phase is being added, the stirrer produces enough shear stress to break up the droplets and form the emulsion. Once all the dispersed phase has been added, the emulsion is stirred at the same rate for a further 5 minutes in order to homogenize the emulsion and ensure the incorporation of all the dispersed phase. The dispersed phase is added through a peristaltic pump (ISMATEC Reglo used in small scale and ISMATEC MCP used in both medium and large scales) to regulate the flow rate. A thermostatic bath (HAAKE F6-C35 used in both small and large scale; HUBER Ministat 230, in medium scale) regulates the temperature of the refrigeration fluid (mixture of Milli-Q water and ethylene glycol) at $25^{\circ} \mathrm{C}$. The digital laboratory stirrer IKA Eurostar power control-visc sets the stirring rate.

The characteristic dimensions of the three scales are shown in Table 1. The linear geometric relation between the three scales is 1:2:4 for both the vessel diameter $(B)$ and height $(H)$. However, for the impeller diameter $(D)$, the impeller blades of the larger scale had to be shortened to prevent the friction of the metallic blades on the vessel glass walls, caused by the increased vibration of the system when working at high stirring rates. Although we do not 
believe that this change significantly alters the results or conclusions, this change should be taken into consideration in the assessment of possible errors and deviations, since variations in the impeller design or diameter can cause significant differences in power consumption or flow patterns $[20,21]$.

Table 1. Characteristic dimensions of the three preparation scales.

\begin{tabular}{|c|c|c|c|c|c|c|c|c|c|}
\hline Scale & $\boldsymbol{B}(\mathbf{c m})$ & $\boldsymbol{H}(\mathbf{c m})$ & $\boldsymbol{D}(\mathbf{c m})$ & $\boldsymbol{D} / \boldsymbol{B}$ & $\boldsymbol{H} / \boldsymbol{B}$ & $\boldsymbol{B} / \boldsymbol{B}_{\text {small }}$ & $\boldsymbol{H} / \boldsymbol{H}_{\text {small }}$ & $\boldsymbol{D} / \boldsymbol{D}_{\text {small }}$ & $\boldsymbol{V}(\mathbf{m L})$ \\
\hline Small & 5 & 4 & 4.5 & 0.9 & 0.8 & 1 & 1 & 1 & 78 \\
\hline Medium & 10 & 8 & 9 & 0.9 & 0.8 & 2 & 2 & 2 & 628 \\
\hline Large & 20 & 16 & 16 & 0.8 & 0.8 & 4 & 4 & 3.6 & 5026 \\
\hline
\end{tabular}

Notation: $B$ : vessel diameter, $H$ : liquid height in the vessel, $D$ : impeller diameter, $V$ : volume of emulsion.

The stirring rate $N(\mathrm{rpm})$ and the addition flow rate $Q(\mathrm{~mL} / \mathrm{min})$ are fixed according to the experimentation plan. The torque $T$ supplied by the agitator was measured along all the process duration. The data were collected in LabWorldSoft (IKA) software.

\subsection{Characterization of the emulsions}

\subsubsection{Rheological parameters}

The rheological tests were performed in a HAAKE Mars III Rheometer (Thermo Fisher Scientifics) and data were collected in HAAKE RheoWin Job Manager and were visualized and saved in HAAKE RheoWin Data Manager. A $35 \mathrm{~mm}$ serrated plate-plate geometry to avoid slippage of the emulsion and with a gap of $0.5 \mathrm{~mm}$ was used. All the tests were performed at $25^{\circ} \mathrm{C}$ (regulated by HAAKE C25-F6 thermostatic bath). Modern rheometers can work in two test modes, controlled stress (CS), in which a controlled stress input $\tau$ is provided and the resulting shear rate $\dot{\gamma}$ is measured or, on the other hand, controlled shear rate (CR), where the rheometer provides a controlled shear rate input and the consequent shear stress is determined. The suitability of each mode depends on the test, as shown in Table 2, which shows the four tests, along with other parameters of the test, and the response variables used. The behavior under flow was analyzed with stress and shear rate steps and oscillatory experiments, to obtain the yield stress $\left(\tau_{0}\right)$ (test \#1), and the steady state viscosity $(\eta)$ dependence on shear rate $(\dot{\gamma})$ 
114 (\#2), the dependency of the viscoelastic parameters $G^{\prime}$ and $G$ '” with frequency $(0.01-100 \mathrm{~Hz})$ in

115 the linear viscoelastic region (LVR) (\#3), and the LAOS behavior, determined by the

116 dependency of $G^{\prime}$ and $G$ '” with oscillatory stress (\#4).

Table 2. Rheological tests for the characterization of the emulsions.

\begin{tabular}{|c|c|c|c|c|c|c|c|}
\hline Test \# & Type of test & Mode & $\begin{array}{c}\text { Measured } \\
\text { variable }\end{array}$ & $\begin{array}{c}\text { Range of } \\
\text { independent variable }\end{array}$ & $\begin{array}{c}\text { Fixed } \\
\text { variable }\end{array}$ & $\begin{array}{c}\text { Steps/step } \\
\text { duration }\end{array}$ & $\begin{array}{c}\text { Response } \\
\text { variable }\end{array}$ \\
\hline $\mathbf{1}$ & Steady Shear & CS & $\gamma$ & $\tau=0.01-100 \mathrm{~Pa}$ & - & $50 / 10 \mathrm{~s}$ & $\tau_{0}$ \\
\hline $\mathbf{2}$ & Flow curve & $\mathrm{CR}$ & $\eta$ & $\gamma=0.001-100 \mathrm{~s}^{-1}$ & - & $30 / 60 \mathrm{~s}$ & $\eta$ \\
\hline $\mathbf{3}$ & Frequency Sweep & $\mathrm{CS}$ & $\mathrm{G}^{\prime}, \mathrm{G}^{\prime \prime}$ & $\omega=100-0.01 \mathrm{~Hz}$ & $\tau=1 \mathrm{~Pa}$ & 30 & $G^{\prime}$ \\
\hline $\mathbf{4}$ & Oscillatory Shear & $\mathrm{CS}$ & $\mathrm{G}^{\prime}, \mathrm{G}^{\prime \prime}$ & $\tau=0.1-1000 \mathrm{~Pa}$ & $\omega=1 \mathrm{~Hz}$ & 200 & $G^{\prime \prime}{ }_{\max } \tau_{c}$ \\
\hline
\end{tabular}

Notation: shear rate $(\dot{\gamma})$, storage modulus $\left(G^{\prime}\right)$, loss modulus $\left(G^{\prime}\right)$, viscosity $(\eta)$, shear stress $(\tau)$, oscillating frequency $(\omega)$, yield

stress $\left(\tau_{0}\right)$, critical stress $\left(\tau_{c}\right)$.

120 The choice of the response variables is based on the rheological behavior of these emulsions.

121 They behave like non-Newtonian plastic fluids, so they undergo creep flow up to a certain value

122 of stress, the yield stress $\tau_{0}$, from which the emulsions flow. With the steady shear test this value

123 is obtained from the shear rate versus shear stress log-log plot as the inflection point in which a

124 large increase in shear rate is observed when varying the shear stress, as also described in other

125 studies [10,22]. Specifically, the yield stress is taken from the intersection point of these two

126 segments (Fig. 9). This steep change in the shear rate value represents the transition from the

127 elastic linear regime to the viscous regime, but could also indicate fracturing of the emulsion structure. Regarding the viscosity, as the emulsions exhibit shear thinning, the viscosity at a shear rate of $1 \mathrm{~s}^{-1}$ is taken for comparison between samples and scales. In the LAOS test, $G{ }^{\prime \prime} \max$

130 and $\tau_{\mathrm{c}}$ are taken, since they also represent the transition from the elastic to the viscous domain.

131 Finally, as these emulsions are highly elastic, the storage modulus $\left(G^{\prime}\right)$ does not vary

132 significantly with frequency in the LVR, so an average value can be obtained, which is used to

133 compare the solid-like behavior of the different emulsions prepared. 


\subsubsection{Droplet size and stability}

As in [7], droplet size and size distribution were determined by optical microscopy (Optika).

137 Samples were prepared by placing a small drop of the emulsion on a glass slide. Droplets were

138 counted on different micrographs (camera Moticam 2300) using Motic Images Plus 2.0 software. The stability of the emulsions was determined by studying the variation of the backscattering (BS) light $\left(173^{\circ}\right)$ using a Turbiscan Classic MA 2000. Moreover, by analyzing the variation of the BS profiles over time, an idea of the destabilization mechanisms is provided. To normalize the BS values and compare them independently from their initial values, the relative BS after 30 minutes and 24 hours was calculated as: $B S_{r e l}=B S_{t} / B S_{t 0}$.

\subsection{Design of experiments at low and medium scales}

In order to study the effect of the process variables on the emulsion rheological properties, the same experimental designs used in [7] were studied. They consist of central composite designs (CCD) formed by factorial, center and star experiments (Fig. 2, blue points). These types of experimental designs are performed at the small and medium scale. The factors varied are the same as in [7]: the stirring rate $(N)$ and the dispersed phase addition flow rate $(Q)$, as preparation variables, and the surfactant-to-oil ratio $(S / O)$, as composition variable. As the final volume of emulsion changes from one scale to another, instead of the addition flow rate $(Q)$, the total addition time $(t=V / Q)$ is taken as variable. The dispersed phase concentration is kept constant at $90 \mathrm{wt} \%$. As there are three factors and two levels, there are in total 16 experiments per scale ( $2^{3}$ factorial experiments, 2 center points, 6 star points). At the medium scale, two replicates of the experiments were performed. The levels of the factors (low and high) were chosen to be the that the scale invariants of these systems were the composition (the surfactant concentration expressed as $S / O$ ), the total addition time $t$ (since it is related to concentration gradients in the system) and the stirring rate $N$. So we would directly know if these were, indeed, the scale invariants, since the same emulsion would be obtained at the different scales. 


\begin{tabular}{|c|c|c|c|}
\hline Factors & Low & High & Center \\
\hline$S / O(\mathrm{wt} / \mathrm{wt})$ & 0.177 & 0.357 & 0.267 \\
\hline$N(\mathrm{rpm})$ & 700 & 1400 & 1050 \\
\hline$t(\mathrm{~min})$ & 8.75 & 3.5 & 5 \\
\hline$Q(\mathrm{~mL} / \mathrm{min})(\mathrm{small})$ & 8 & 20 & 14 \\
\hline$Q(\mathrm{~mL} / \mathrm{min})($ medium $)$ & 64 & 160 & 112 \\
\hline
\end{tabular}

162

163 The procedure to obtain the empirical models is described in our previous study. Basically, a

164 statistical analysis with Statgraphics Plus software is performed, in which the significance of the

165 factors is given with a $95 \%$ confidence interval. With the Pareto chart the significant factors are

166 visualized, which are the ones that appear in the empirical models derived, from which the

167 response surfaces are created. These surfaces are useful to see the variation of the studied

168 parameters with the factors and detect the interactions between factors.

169 Once the models are obtained, a validation process is carried out performing new experiments

170 within the experimental range. Simple experimental designs (Fig. 2, green lines), in which only

171 one factor is changed while the others remain constant, are developed so as to extend the

172 number of experiments and to contrast the model predictions with the experimental results.
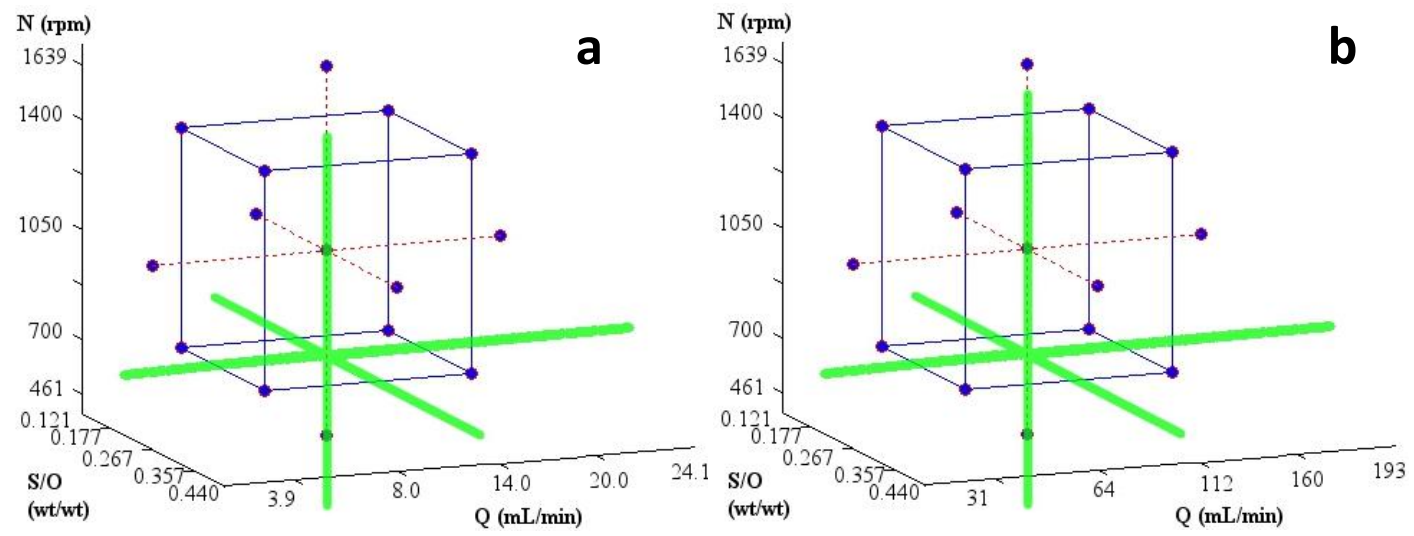

Fig. 2. Graphical representation of the CCD (blue points) and validation experiments (green thick lines) at (a) small and (b) medium scales.

These experiments are performed in three blocks: 

$S / O=0.267 \pm 0.020$ and the addition time $t$ at $5 \mathrm{~min} ;$ $S / O=0.267 \pm 0.020 ;$ and

The three simple designs can be observed as green lines in Fig. 2-a for small scale and Fig. 2-b for medium scale. Only in the conditions of block (1) were experiments from the CCD performed. In blocks (2) and (3) the validation experiments were compared directly with the model, since no CCD experiments were performed in those conditions, as observed in the corresponding plots.

The empirical models obtained from the CCD experiments were used to obtain a first approximation of the scale invariants, which was then completed by adding the validation experiments. Next, some experiments at the large scale were carried out and were used, together with all the other experiments, to obtain a general power law exponent, as we will see in further sections.

\subsection{Experiments at large scale}

To obtain a reliable scale-up invariant, experiments at the large scale were also performed. If the scale-up model obtained with the small and medium scale is validated using the large scale vessel, it would mean that the model can be applied to many different scales, other than the ones used in this study to obtain the model. Due to the high amount of product needed for the large scale (even if it is $90 \mathrm{wt} \%$ water, nearly $500 \mathrm{~mL}$ of dodecane are needed for every experiment) the minimum number of experiments was done. The experiments performed at large scale are shown in Table 4. Basically, the stirring rate $N$ was varied from 263 to $788 \mathrm{rpm}$ at a fixed $S / O=0.267$ and $t=5.6 \min (\exp 1-4)$, and then two more stirring rates at $S / O=0.42$ and two different times $t$ were tested. In the large scale installation, experiments at very high stirring rates could not be performed, because the stirrer could not rotate the impeller due to the high 
amount of highly-viscous emulsion formed. The maximum torque provided for the stirrer was $90 \mathrm{~N} \cdot \mathrm{cm}$, so when the torque required to stir the emulsion was higher, the stirring rate was reduced automatically. In fact, in experiment \#4, at $788 \mathrm{rpm}$, after $3.75 \mathrm{~min}$, the torque reached its maximum value and immediately after, the $N$ decreased sharply, while the torque remained at $80 \mathrm{~N} \cdot \mathrm{cm}$ during the rest of the experiment. This experiment is not taken into account for the scale-up model but is useful to show here.

Table 4. Experiments performed at large scale.

\begin{tabular}{|c|c|c|c|c|}
\hline Exp. \# & $\begin{array}{c}\mathbf{S} / \mathbf{O} \\
(\mathbf{w t} / \mathbf{w t})\end{array}$ & $\begin{array}{c}\boldsymbol{Q} \\
(\mathbf{m L} / \mathbf{m i n})\end{array}$ & $\begin{array}{c}\boldsymbol{T} \\
(\mathbf{m i n})\end{array}$ & $\begin{array}{c}\boldsymbol{N} \\
(\mathbf{r p m})\end{array}$ \\
\hline 1 & 0.267 & 895 & 5.6 & 263 \\
\hline 2 & 0.267 & 895 & 5.6 & 394 \\
\hline 3 & 0.267 & 895 & 5.6 & 525 \\
\hline 4 & 0.267 & 895 & 5.6 & $788^{\star}$ \\
\hline 5 & 0.42 & 895 & 5.6 & 200 \\
\hline 6 & 0.42 & 248 & 20.3 & 400 \\
\hline
\end{tabular}

*Only up to minute 3.75 , then $\mathrm{N}$ decreased due to the lack of power input to keep the stirring for the large amount of

\section{Results and discussion}

\subsection{Empirical models obtained from the CCD: influence of the process} variables on the rheological features of emulsions and model

In [7], we obtained the empirical models at small and medium scale for the droplet size and polydispersity. Here, following the same procedure, we derive the empirical models for the rheological parameters (Table 5). The ones chosen are the yield stress $\tau_{0}$, the storage modulus $G^{\prime}$ (taken as an average value of the plateau value between 0.01 and $10 \mathrm{~Hz}$ in the oscillatory

221 test), the viscosity $\eta$ at $1 \mathrm{~s}^{-1}$, the critical stress $\tau_{c}$, and the value reached by the loss modulus at 222 the critical stress point $G{ }^{\prime}{ }_{\max }$. 
The empirical models include the significant factors that have an influence on each emulsion

224 feature, which are the ones whose p-value is less than 0.05 , indicating that they are significantly

225 different from zero at the $95 \%$ confidence level (Fig. 3-4 show an example for $G$ ' ${ }_{\max }$ at both

226 scales). From the models, the response surfaces are obtained (e.g. Figs. 5-8), and the effect of

227 the factors on the rheological properties is clearly seen. Moreover, the values at small and

228 medium scale can be compared. As the experiments were performed in the same range of $N$,

$229 S / O$, and $t$, the values for small scale are usually lower than the values obtained at the same

230 conditions at medium scale. For example, if we compare the response surfaces for the yield

231 stress at both scales (Figs. 7 and 8), for a fixed $S / O=0.17$, at $700 \mathrm{rpm}$, for the medium scale the

232 yield stress is higher than $50 \mathrm{~Pa}$, but a stirring rate higher than $1000 \mathrm{rpm}$ at small scale is needed

233 to obtain the same value. This indicates that these factors are not the proper scale invariants of

234 the process, and that a scale invariant which leads to a higher stirring rate at the small scale is

235 needed, in order to obtain the same final product in all the preparation scales. The rest of the

236 Pareto charts and response surfaces can be found in the supplementary data (SD).

Table 5. Empirical models for the rheological parameters at small and medium scale. Notation and units: $\tau_{0}(\mathrm{~Pa})$ :

\begin{tabular}{|c|c|}
\hline Empirical models at small scale & Empirical models at medium scale \\
\hline$\tau_{0}(\mathbf{P a})=-15.92+86.59 \cdot \mathbf{S} / \mathbf{O}-0.75 \cdot \mathbf{Q}+0.042 \cdot \mathbf{N}$ & $\tau_{0}(\mathrm{~Pa})=-28.96+45.66 \cdot \mathrm{S} / \mathrm{O}-0.118 \cdot \mathrm{N}-3.3 \cdot 10^{-5} \cdot \mathrm{N}^{2}$ \\
\hline $\mathbf{G}^{\prime}(\mathbf{P a})=-293.28+1117.7 \cdot \mathbf{S} / \mathbf{O}+0.357 \cdot \mathbf{N}$ & $\begin{array}{c}\mathbf{G}^{\prime}(\mathbf{P a})=-546.75+2327.03 \cdot \mathbf{S} / \mathbf{O}-0.851 \cdot \mathbf{Q}+ \\
1.208 \cdot \boldsymbol{N}-1.59 \cdot \mathbf{S} / \mathbf{O} \cdot \mathbf{N}-9.53 \cdot 10^{-5} \cdot \mathbf{N}^{2}\end{array}$ \\
\hline $\begin{array}{c}\mathbf{G}_{\max }(\mathrm{Pa})=-54.46+188.20 \cdot \mathbf{S} / \mathbf{O}-0.901 \cdot \mathbf{Q}+ \\
0.103 \cdot \mathbf{N}\end{array}$ & $\begin{array}{c}\mathbf{G}_{\max }(\mathrm{Pa})=-82.36+137.98 \cdot \mathbf{S} / \mathbf{O}-0.1143 \cdot \mathbf{Q}+ \\
0.2953 \cdot \boldsymbol{N}-2.8 \cdot 10^{-5} \cdot \boldsymbol{N}^{2}\end{array}$ \\
\hline$\tau_{c}(P a)=-45.51+151.1 \cdot S / O+0.063 \cdot N$ & $\tau_{c}(P a)=-59.80+94.92 \cdot S / O+0.194 \cdot N-5.6 \cdot 10^{-5} \cdot N^{2}$ \\
\hline $\begin{array}{c}\eta\left(\mathbf{1 ~ s}^{-1}\right)(\mathbf{P a} \cdot \mathbf{s})=16.79+99.77 \cdot \mathbf{S} / \mathbf{O}-2.45 \cdot \boldsymbol{Q}+ \\
0.012 \cdot \boldsymbol{N}+0.0018 \cdot \boldsymbol{Q} \cdot \boldsymbol{N}\end{array}$ & $\begin{array}{c}\eta\left(1 \mathbf{~ s}^{-1}\right)(\mathbf{P a} \cdot \mathbf{s})=-49.43+95.19 \cdot \mathbf{S} / \mathbf{O}+0.143 \cdot \mathbf{N}- \\
4.74 \cdot 10^{-5} \cdot \mathbf{N}^{2}\end{array}$ \\
\hline
\end{tabular}




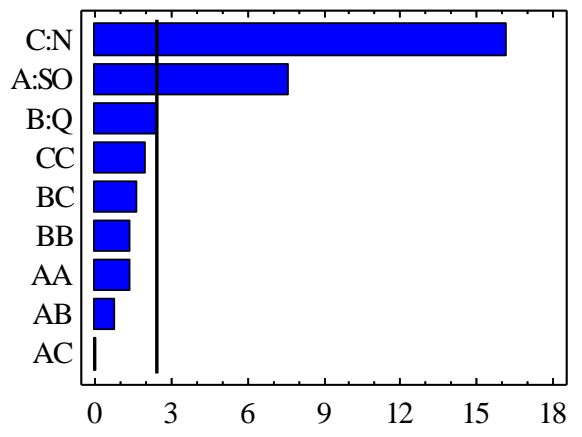

Standardized effect for G'max

Fig. 3. Pareto chart for $G^{\prime}{ }^{\max }$ showing the significant effects for small scale.

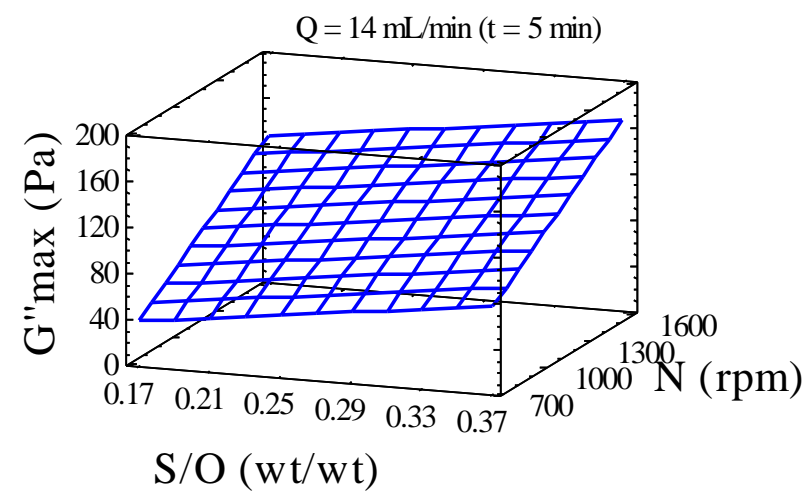

Fig. 5. Response surface for $G^{\prime \prime}{ }_{\max }$ at small scale as a function of $\mathrm{S} / \mathrm{O}$ and $\mathrm{N}$.

242

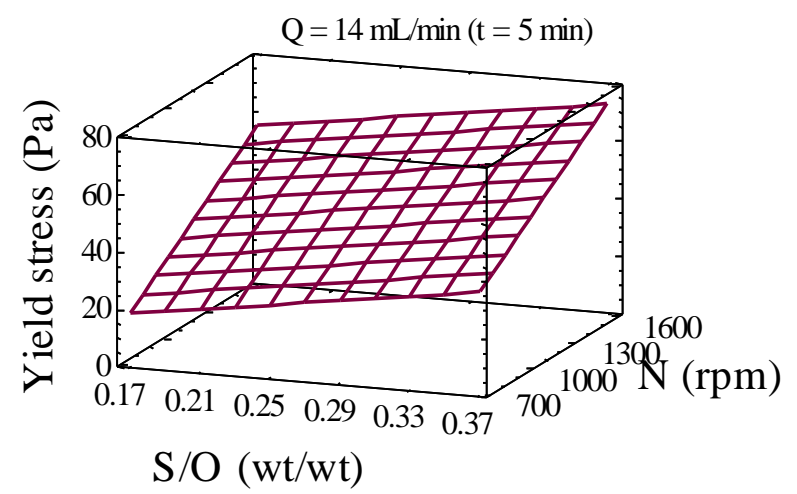

Fig. 7. Response surface for yield stress $\tau_{0}$ at small scale as a function of $\mathrm{S} / \mathrm{O}$ and $\mathrm{N}$.

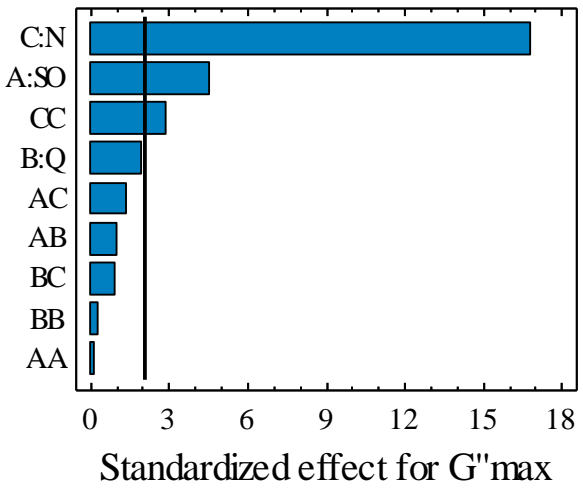

Fig. 4. Pareto chart for $G^{\prime \prime}{ }_{\max }$ showing the significant effects for medium scale.

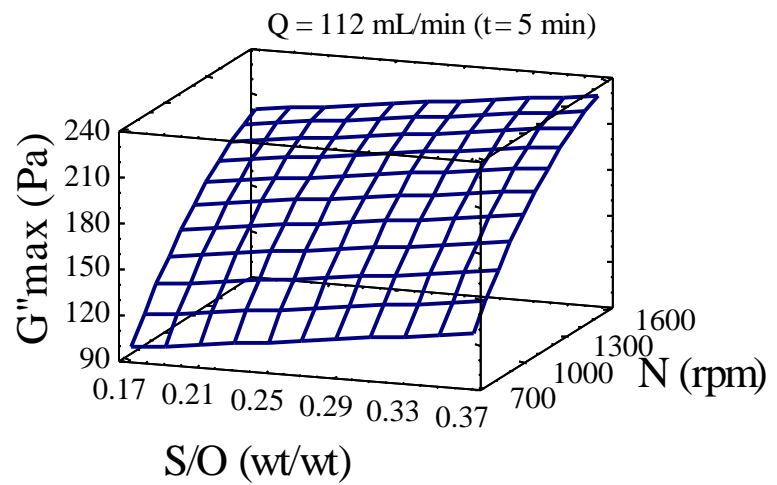

Fig. 6. Response surface for $G{ }^{\prime}{ }_{\max }$ at medium scale as a function of S/O and $\mathrm{N}$.

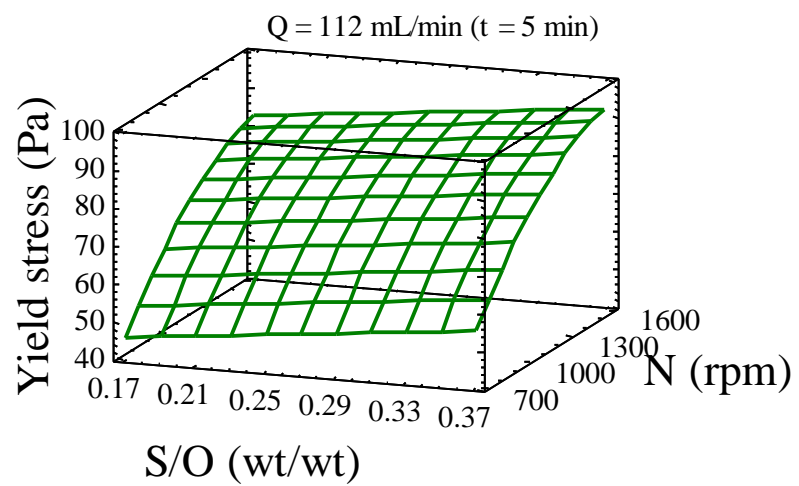

Fig. 8. Response surface for yield stress $\tau_{0}$ at medium scale as a function of $\mathrm{S} / \mathrm{O}$ and $\mathrm{N}$.

\subsubsection{Effect of stirring rate}

244 According to the statistical analysis, the factor with the greatest influence on the rheological

245 parameters in the range studied is the stirring rate. In all the Pareto charts, this is the first factor 
to appear. The influence of this factor appears to be lineal in the experiments at small scale,

247 whereas it has a quadratic effect on the experiments at medium scale. Its effect is positive for all

248 the rheological parameters: the higher the stirring rate, the higher the values of the parameters

249 (e.g. Figs. 5-8). The effect of the stirring rate $N$ on the emulsion rheological behavior is due to

250 the increase of the energy input, which leads to an increased droplet breakup, which involves

251 the formation of smaller emulsion drops. This stored energy is translated into a more elastic

252 behavior of the emulsion. This is reflected on the increase of the yield stress $\tau_{0}$, as observed in

253 Figs. 7 and 8 and also in Fig. 9, where the shear rate variation with shear stress is represented

254 for samples at different $N$, showing the increase of the yield stress when $N$ increases. The

255 critical yield stress $\tau_{\mathrm{c}}$, the viscosity and the storage modulus also increase with $N$. For example,

$256 G^{\prime}$ rises considerably from $200 \mathrm{~Pa}$ to $1000 \mathrm{~Pa}$ when the stirring rate is increased from $350 \mathrm{rpm}$

257 to $1600 \mathrm{rpm}$ at the medium scale (at fixed $S / O=0.267$ and $t=5 \mathrm{~min}$ (Fig. 12)).

258

259

260

261

262

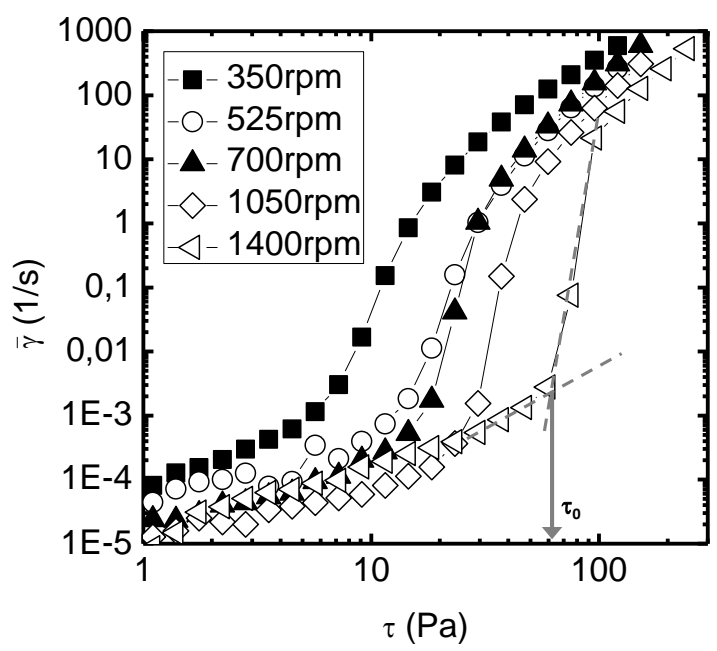

Fig. 9. Steady shear test at equal $S / O=0.267$ and $t=5 \mathrm{~min}$ at small scale. The stirring rate $N$ varies from $350 \mathrm{rpm}$ to $1400 \mathrm{rpm}$.

\subsubsection{Effect of surfactant-to-oil ratio}

The second factor that has an influence is the surfactant concentration, in terms of surfactant-tooil ratio $S / O$. This term appears in every empirical model and has a positive effect. An increase of the surfactant concentration $S / O$ in the emulsion produces a stronger solid-like behavior. As explained in [7], the more surfactant, the more interfacial area can be stabilized and the longer 
the time that the smaller droplets can remain stable without experiencing coalescence, which involves a more compact droplet network and increased elastic behavior. As observed in Figs. 7 and 8, the yield stress $\tau_{0}$ increases approximately $10 \mathrm{~Pa}$ when $S / O$ is raised from 0.17 to 0.37 , at both scales.

\subsubsection{Effect of addition time}

The dispersed phase addition flow rate $Q$, or addition time $t$, has less influence on the emulsion properties than the stirring rate $N$ and the surfactant-to-oil ratio $S / O$. However, this factor has a slight influence on the rheological parameters, especially at short times. Although the difference in the rheological parameters at the lowest and highest level of the time range tested is small, focusing on the yield stress $\tau_{0}$ and on the critical yield stress $\tau_{c}$, their values are lower at high addition flow rate $Q$ (low addition time $t$ ) (e.g. Fig. 11). This is because a decrease in the addition time decreases the elastic behavior of the emulsion, since there is less time to break up the droplets and. Hence, they occupy larger domains and have less elastic energy stored. When the energy input (stirring rate $N$ ) is constant, if the addition flow rate is less (addition time $t$ high), the ratio between energy used to disperse the water and the amount of water is higher, so the droplets of the dispersed phase are finer because more energy can be used to break up the large droplets added. Therefore, increasing the addition flow rate $Q$ or reducing the addition time $t$ implies a slightly decrease of the solid-like behavior of the emulsion. For example, at small scale, below $Q=10 \mathrm{~mL} / \mathrm{min}$, the properties of the emulsion do not differ significantly with the variation of the addition flow rate, but at higher $Q$, the properties slightly change, albeit less so than with the stirring rate or concentration of surfactant.

\subsubsection{Validation of the models}

The validation of the models and the verification of the parameters influence on the emulsion properties is carried out at both scales (small and medium) following simple designs, varying one variable and maintaining the other two constant (blocks 1, 2 and 3), and performing from 5 to 7 experiments in each case. 
292 The results are similar for both scales, and some examples (small scale: Figs. 10 and 11;

293 medium scale: Figs. 12 and 13) are depicted for discussion. The rest of the figures are found in

294 the SD. For $\tau_{0}$ and $\tau_{c}$, the experimental values are somewhat lower than the model predictions,

295 although they do follow the same pattern: increase with $N$ and $S / O$ and decrease with $Q$. They

296 seem to fit the model at low-medium stirring rates, when varying this variable (Fig. 10). The

297 storage (Fig. 12) and the loss modulus are the response variables that best fit the model

298 predictions, for all the three variables studied. When varying $N, S / O$ or $Q$, the experimental

299 values are found randomly following the same behavior as the model in the range studied. The

300 dispersion is higher for the $G{ }^{\prime}{ }_{\text {max }}$ values, but, in general, the values are of the same order. In

301 general, the viscosity follows the same behavior as the model predictions, although the values

302 are also lower than expected (Fig. 13).

303 A possible explanation of the discrepancy between the model predicted values and the

304 experimental data is the fact that the validation experiments were performed in some regions

305 where no experimental points were done previously. CCD experiments were performed in the

306 validation simple design only when the stirring rate is varied (Fig. 2). When varying the $S / O$ and

$307 Q$, although the values are in the range studied, there are no experiments in that region. This

308 may explain why the validation of the model when varying the stirring rate is better than when

309 varying the other two variables, since the empirical model was obtained, in this latter case, from

310 experiments in other regions. Nevertheless, the behavior of the rheological parameters and the

311 variation with the factors is the same, although the values are lower than the predictions. 


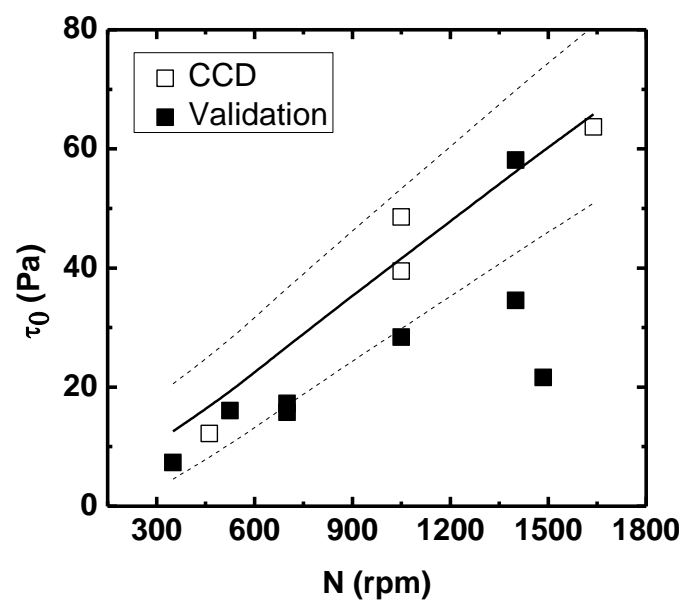

Fig. 10. Variation of $\tau_{0}$ with stirring rate at small scale.

Experiments from CCD and validation. Line: model; dashed lines: confidence interval.

312

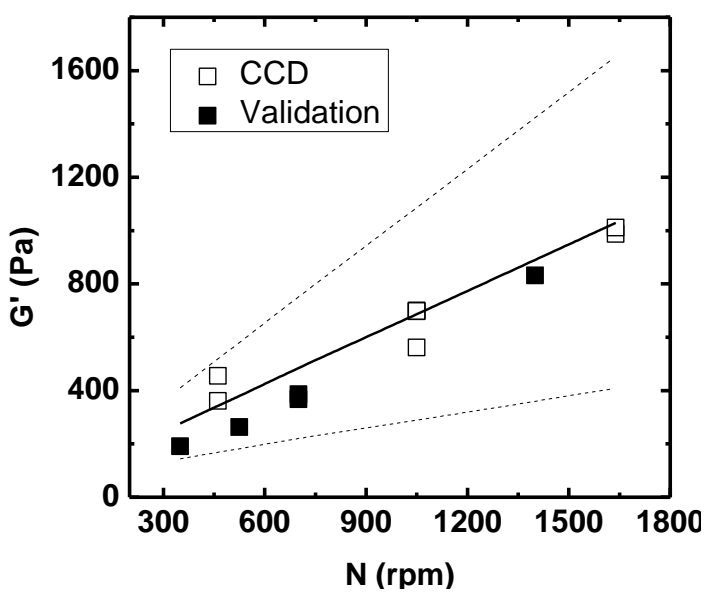

Fig. 12. Variation of $G$ ' with stirring rate at medium scale. Experiments from CCD and validation. Line: model; dashed lines: confidence interval.

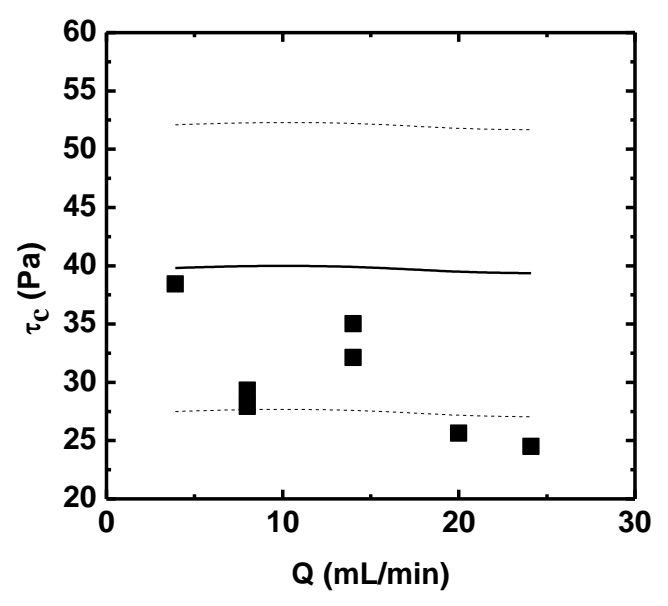

Fig. 11. Variation of $\tau_{\mathrm{c}}$ with dispersed phase addition flow rate at small scale. Validation experiments only. Line: model; dashed lines: confidence interval.

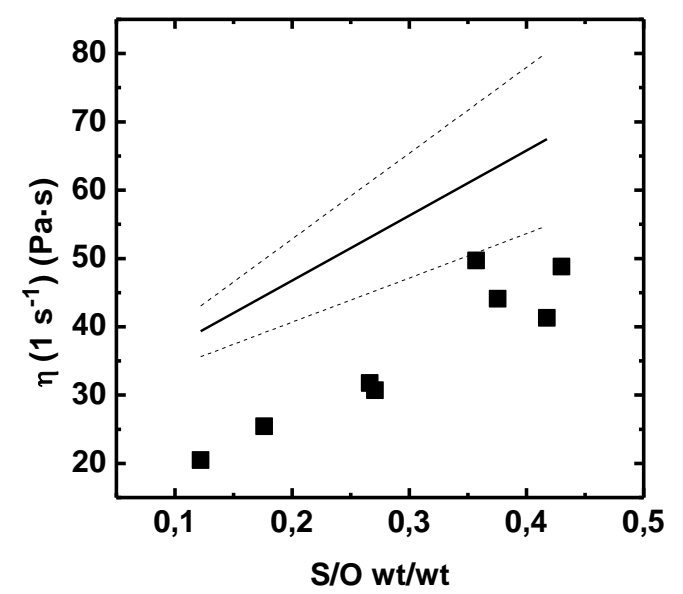

Fig. 13. Variation of $\eta\left(1 \mathrm{~s}^{-1}\right)$ with $S / O$ at medium scale. Validation experiments only. Line: model; dashed lines: confidence interval.

315 As discussed in [7], the scale-up invariants are the composition of the emulsion (the dispersed

316 phase concentration and the $S / O$ ratio), the addition time of dispersed phase $(t)$ and, regarding

317 the agitation rate and preparation scale, the parameter $N D^{\alpha}$, where $N$ is the stirring rate and $D$

318 the impeller diameter, so the power law exponent, $\alpha$, has to be derived from the data. The first 
approximation to obtain this parameter is using the empirical models that describe the behavior of the emulsions as a function of the process variables, obtained from the experimental design with the CCD experiments at small and medium scale. Due to the apparent linearity of the results when plotting the models of the two scales together, the method used is based on applying a linear regression between the rheological parameters and $N D^{\alpha}$, where $\alpha$ is optimized finding the highest correlation coefficient for all the points. The form of the regression is (1):

Where $N D^{\alpha}$ is defined as $N D^{\alpha}\left(\mathrm{cm}^{\alpha} / \mathrm{s}\right)=\pi \cdot N\left(\min ^{-1}\right) / 60 \cdot \mathrm{D}^{\alpha}\left(\mathrm{cm}^{\alpha}\right)$, to be consistent with the units usually given to the tip speed $(v=N D$, in $\mathrm{cm} / \mathrm{s})$. Due to equipment limitation, the results at high $N D^{\alpha}$ (when $N>1050 \mathrm{rpm}$ ) are very disperse and do not fit conveniently with any linear regression, so these experiments are not included in the range in which the following scale-up model is applied. The power law exponent is determined in all the possible combinations of $S / O$ and addition time $t$. However, for the sake of clarity, the conditions chosen to present in this study are the same conditions chosen for the validation experiments, so that a further comparison can be easier and clearer. As said previously, the model validation experiments were carried out fixing two different surfactant concentration $S / O(S / O=0.27 \pm 0.02$ and $S / O=0.43 \pm 0.02)$ and two different addition times $t(t=5.08 \mathrm{~min}$ and $t=18.24 \mathrm{~min})$ at each scale. The combinations of the levels of these two factors are shown in Table 6. We will refer to them as conditions \#1 $(S / O=0.27$ and $t=5.08 \mathrm{~min}), \# 2(S / O=0.43$ and $t=5.08 \mathrm{~min})$, and \#3 $(S / O=0.43$ and $t=18.24 \mathrm{~min})$ from now on.

\begin{tabular}{|c|c|c|}
\hline Conditions \# & $\mathbf{S} / \mathbf{O}(\mathbf{w t} / \mathbf{w t})$ & $\mathbf{t}(\mathbf{m i n})$ \\
\hline 1 & $0.27 \pm 0.02$ & 5.08 \\
\hline 2 & $0.43 \pm 0.02$ & 5.08 \\
\hline 3 & $0.43 \pm 0.02$ & 18.24 \\
\hline
\end{tabular}


The values from each model were obtained fixing a significant number of levels of $N$ (included

342 in the experimental range) and then $\alpha$ was optimized by maximizing the correlation coefficient

343 of all them to the linear equation presented previously. The results are shown in Table 7 and the

344 regression coefficients in the SD. Each rheological parameter has a different power law

345 exponent $\alpha$. However, as the behavior of the emulsions is defined by all the parameters, a

346 general power law exponent $\alpha$ defined from the five rheological parameters is found by

347 maximizing the sum of the correlation coefficients. In Table 7, first the exponent for each of the

348 five rheological parameters chosen is shown, and in the last column, the exponent obtained from

349 all the rheological parameters together. These values are in the range from 0.55 to 0.71 , so they

350 agree with [7], in which we obtained the scale invariant from the droplet size models, and found

351 that it approached 0.5 for small stirring rates $N$. In that previous study, it was observed that $\alpha$

352 approached zero when $N$ was very high, but in this study we have focused on values below

$353 N=1050 \mathrm{rpm}$, due to the experimental equipment limitation.

Table 7. Power law exponent $\alpha$ from general models at small and medium scale.

\begin{tabular}{|c|c|c|c|c|c|c|}
\hline Conditions & $\begin{array}{l}\text { Storage } \\
\text { modulus }\end{array}$ & $\begin{array}{c}\text { Loss } \\
\text { modulus }\end{array}$ & $\begin{array}{c}\text { Critical } \\
\text { yield stress }\end{array}$ & $\begin{array}{l}\text { Yield } \\
\text { stress }\end{array}$ & $\begin{array}{l}\text { Viscosity } \\
\text { at } \dot{\gamma}=1 \mathrm{~s}^{-1}\end{array}$ & $\begin{array}{l}\text { Rheological } \\
\text { parameters }\end{array}$ \\
\hline 1 & 0.81 & 0.90 & 1.06 & 0.73 & -0.07 & 0.71 \\
\hline 2 & 1.05 & 0.72 & 0.60 & 0.52 & 0.04 & 0.55 \\
\hline 3 & 1.57 & 0.80 & 0.35 & 0.35 & -0.01 & 0.57 \\
\hline
\end{tabular}

3.2.2 Lineal scale-up models at fixed conditions using the validation experimental values at small, medium and large scales

The experiments performed for the model validation were also used to determine the power law exponent $\alpha$. This was done using the same method as in the previous part, where we obtained it from the empirical models derived from the CCD experiments. In this case, though, the regression was done with experiments on the three scales: small, medium and large. Table 8

361 shows the power law exponents obtained and in the SD the model parameters and regression coefficients are found for every condition and parameter. The power law exponent is found 

between 0.49 and 0.62 , with an average value of $0.55 \pm 0.07$.

Table 8. Power law exponent for each rheological parameter and for the combination of all them.

366

\begin{tabular}{|c|c|c|c|c|c|c|}
\hline Conditions & $\begin{array}{c}\text { Storage } \\
\text { modulus }\end{array}$ & $\begin{array}{c}\text { Loss } \\
\text { modulus }\end{array}$ & $\begin{array}{c}\text { Critical } \\
\text { yield stress }\end{array}$ & $\begin{array}{c}\text { Yield } \\
\text { stress }\end{array}$ & $\begin{array}{c}\text { Viscosity } \\
\text { at } \dot{\gamma}=1 \mathbf{s}^{-1}\end{array}$ & $\begin{array}{c}\text { Rheological } \\
\text { parameters }\end{array}$ \\
\hline 1 & 0.67 & 0.63 & 0.51 & 0.43 & 0.34 & 0.53 \\
\hline 2 & 0.62 & 0.59 & 0.44 & 0.51 & 0.32 & 0.49 \\
\hline 3 & 0.75 & 0.61 & 0.56 & 0.57 & 0.62 & 0.62 \\
\hline
\end{tabular}

Comparing the values obtained from the empirical models at each scale (Table 7) with those obtained from the validation experimental points (Table 8), only in a few occasions both are similar. Despite this fact, when the power law exponent $\alpha$ is calculated maximizing the sum of the five rheological parameters correlation coefficients, the values are really close: $0.61 \pm 0.09$ and $0.55 \pm 0.07$. Moreover, both power law exponent $\alpha$ (from models and experiments) possess the same behavior when the conditions change. Remaining constant the addition time $t$ (conditions \#1 and \#2), the exponent $\alpha$ decreases in most of the rheological values when $S / O$ decreases - and also in the general exponent. On the other hand, at constant surfactant amount $S / O$ (conditions \#2 and \#3), the power law exponent $\alpha$ slightly increases its value when the addition time $t$ is raised.

In the $\mathrm{CCD}$, the experiments are performed at different factor levels, so there are not many experiments at the same conditions of $S / O$ and $t$ : taking into account the experiments at small and medium scale, there are three experiments at conditions \#1, only one experiment at conditions \#2, and none at conditions \#3. Hence, there are probably a low number of experiments at the same experimental conditions to obtain a reliable power law exponent as described here. Also, we must not forget that it is obtained from the empirical models. Hence, performing more experiments is necessary to ensure the validity of the scale-up invariants. This is why the validation experiments, as they are performed only varying one variable at a time, 
can be used to obtain the power law exponents at some fixed conditions with probably more reliability than the power law exponents obtained from the empirical models of the CCD experiments.

\subsubsection{Specific and global models from all the validation experiments}

Once these models are obtained and the similarity among power law exponents $\alpha$ at different conditions is corroborated, it seems possible to obtain a scale-up model which includes all of the validation experiments and define the value of any rheological parameter as a function of the three scale invariants: surfactant concentration $S / O$, addition time $t(\min )$ and $N D^{\alpha}$ (as a factor which includes the stirring rate $N$, impeller diameter $D$-scale- and the power law exponent $\alpha$ ). The exponent $\alpha$ will be optimized in order to obtain the maximum correlation coefficient (the procedure is the same as explained previously).

Table 9 shows the specific $\alpha$ for each rheological parameter and a global $\alpha$ which is found from the maximization of the sum of correlation coefficients for each rheological parameter. A value of 0.63 is obtained in this case. In the SD, the coefficients for the model parameters and the regression coefficients of the models when applying the specific or the global power law exponent are shown.

Table 9. The power law exponent $\alpha$ which defines the whole experimental system.

\begin{tabular}{|c|c|c|c|c|c|}
\hline $\begin{array}{c}\text { Storage } \\
\text { modulus }\end{array}$ & $\begin{array}{c}\text { Loss } \\
\text { modulus }\end{array}$ & $\begin{array}{c}\text { Critical } \\
\text { yield stress }\end{array}$ & $\begin{array}{c}\text { Yield } \\
\text { stress }\end{array}$ & $\begin{array}{c}\text { Viscosity } \\
\text { at } \dot{\gamma}=1 \mathbf{s}^{-1}\end{array}$ & $\begin{array}{c}\text { Rheological } \\
\text { parameters }\end{array}$ \\
\hline 0.73 & 0.77 & 0.66 & 0.49 & 0.45 & 0.63 \\
\hline
\end{tabular}




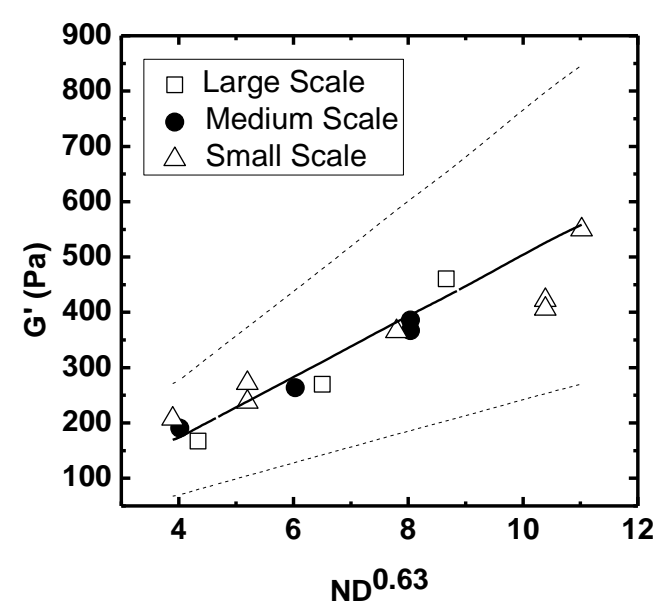

Fig. 14. Representation of the experimental points together with the scale-up model for G'. $(S / O=0.267$, $t=5 \mathrm{~min})$

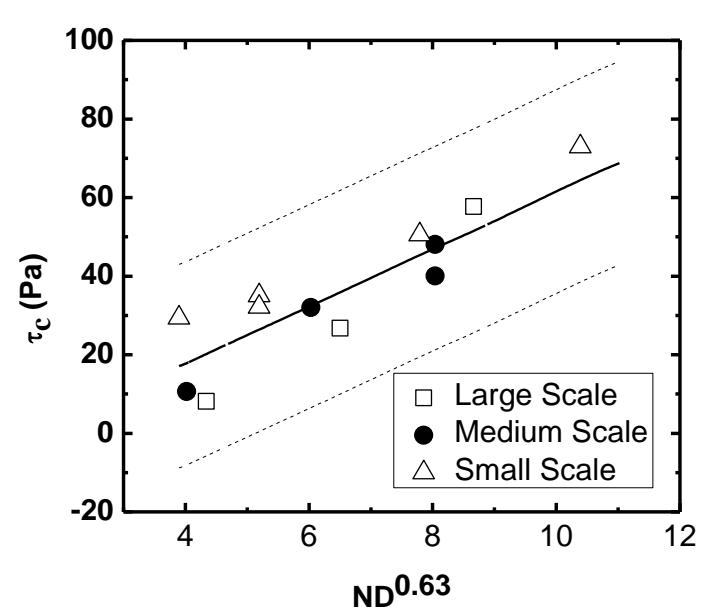

Fig. 15. Representation of the experimental points together with the scale-up model for $\mathrm{G}^{\prime}$. $(S / O=0.267$, $t=5 \mathrm{~min})$

The experimental results are plotted with the scale-up models and, as expected, the model with the specific power law exponent for each variable fits more properly the experimental results, yet the difference with the global model is not that significant. This difference is more remarkable at conditions $\# 1(S / O=0.267 \pm 0.020$ and $t=5.08 \mathrm{~min})$ due to larger number of experiments available, than at the other two conditions, where both models (specific and general) include perfectly (in almost all the parameters) the experimental values. The global models, for example for $G^{\prime}$ and $\tau_{\mathrm{c}}$ (Figs. 14 and 15) clearly fit with the experimental points of

411 the three scales and are clearly inside the $95 \%$ confidence interval. Moreover, the random

412 distribution of the experimental results under and above the model prediction shows the significance of the regression, since no specific behaviors are followed by any scale. 


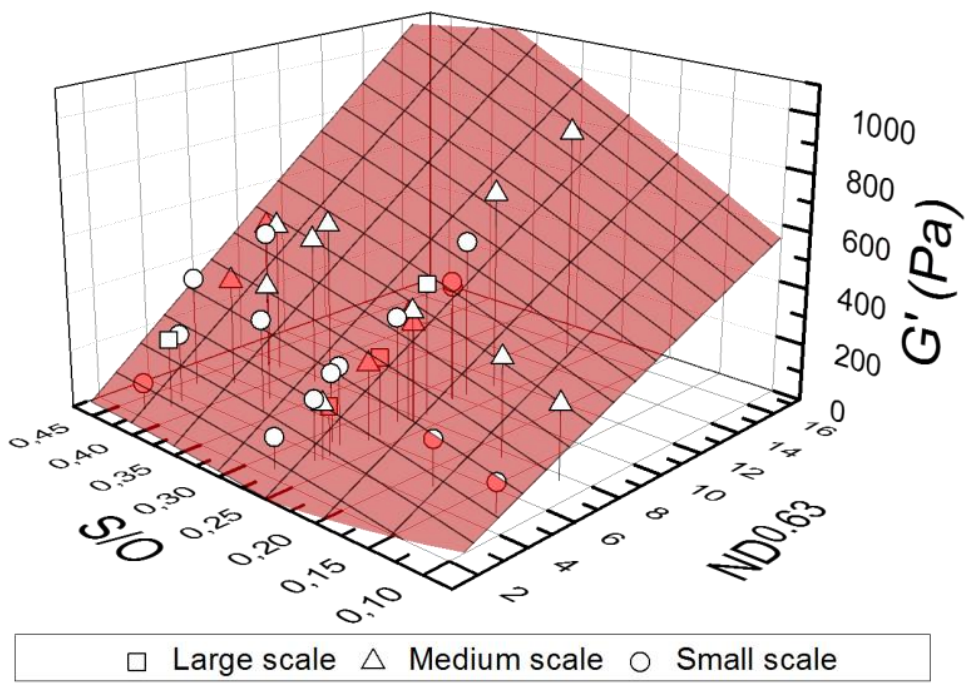

Fig. 16. Scale-up general model $(\alpha=0.63)$ and the validation experiments points at small, medium and large scale $(t=5.08 \mathrm{~min})$ for $G^{\prime}$.

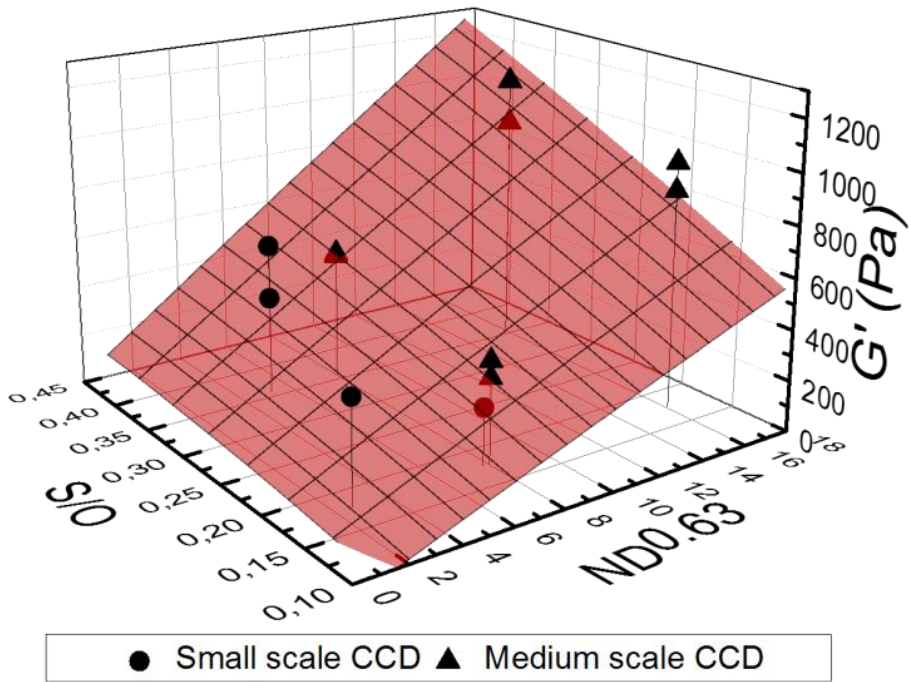

Fig. 17. Scale-up general model $(\alpha=0.63)$ and the CCD experiments points at small and medium scale $(t=8.84 \mathrm{~min})$ for $G^{\prime}$.

415 The next step is the confirmation that the scale-up model obtained from the validation

416 experiments is valid and includes the values from which the models at each scale were

417 calculated. In order to illustrate the possible differences, a response surface graph has been

418 depicted, where the addition time has been fixed and the other two factors $\left(N D^{\alpha}\right.$ and $\left.S / O\right)$ vary

419 along the experimental range. $N D^{\alpha}$ includes the scale -as $D$, impeller diameter-, so it allows us

420 to depict both scales (small and medium) at the same graph. Fig. 16 shows an example at

$421 t=5.08 \mathrm{~min}$ for the storage modulus. The random distribution of points of different scales and

422 the proximity of the points to the model confirms its validity. This behavior is followed by the

423 storage modulus $G^{\prime}$ and the loss modulus $G^{\prime \prime}{ }_{\max }$; for the rest of the rheological parameters, the

424 experimental values remain mainly above the surface. The validity of the models at each scale

425 (where $G^{\prime}$ and $G^{\prime \prime}{ }_{\max }$ fit the models properly) is intrinsically related with these results.

426 Next we investigate if the model obtained from the validation experiments can explain the

427 behavior of the CCD experiments. Fig. 17 shows the graphical representation of all the CCD

428 experimental values (both scales) at $t=8.84$ min with the global model obtained from the

429 validation experiments $(\alpha=0.63)$. The random distribution under and above the response

430 surface indicates the validity of the global model. 


\subsubsection{General models from all the experiments performed in the system: CCD and} validation experiments at three scales

433 Confirmed the similarity between the CCD experiments and validation experiments, we then correlate all experiments at the three scales (CCD and validation) with a unique quadratic model for each rheological parameter, as a function of the three scale invariants: surfactant concentration $S / O$, addition time $t$ and $N D^{\alpha}$ (stirring rate $N$ and scale), following the same procedure as before -maximization of the sum of the correlation coefficients-, thus, broadening the application range of the model. The power law exponent found for this general model is $\alpha=0.65$, and is close to that obtained with only the validation experimental values ( $\alpha=0.63$ ), which confirms the similarity of behavior between all the emulsions prepared. A random distribution of values of different scales along the experimental range is shown in $G^{\prime}$ (Fig. 18) and $G{ }^{\prime}{ }_{\max }$ models, while for the other parameters, due to the difference of the value -not of the behavior- in some fixed conditions, the experimental values of a scale remain only under or above the model prediction.

Therefore, the validation experiments fit the global model derived from them, and the difference between them and the general model from all the experiments is not significant. The divergence observed in the small and medium scale validation is minimized and confirms, as discussed in the previous section, the validity of the models. The difference in the impeller diameter at large scale does not seem to bring unexpected results, in terms of the emulsion properties.

Fig. 18 depicts the response surface obtained with the general model $(\alpha=0.65)$ and all the experiments at $t=5.08 \mathrm{~min}$, and Fig. 19, the general model $(\alpha=0.65)$ at (a) $t=18.24 \mathrm{~min}$ and $S / O=0.43 \pm 0.02$ and (b) $t=5.08 \mathrm{~min}$ and $S / O=0.43 \pm 0.02$. The experimental values follow a random distribution around the model prediction. The model parameters, regression coefficients and figures for the five rheological parameters are found in the SD. 


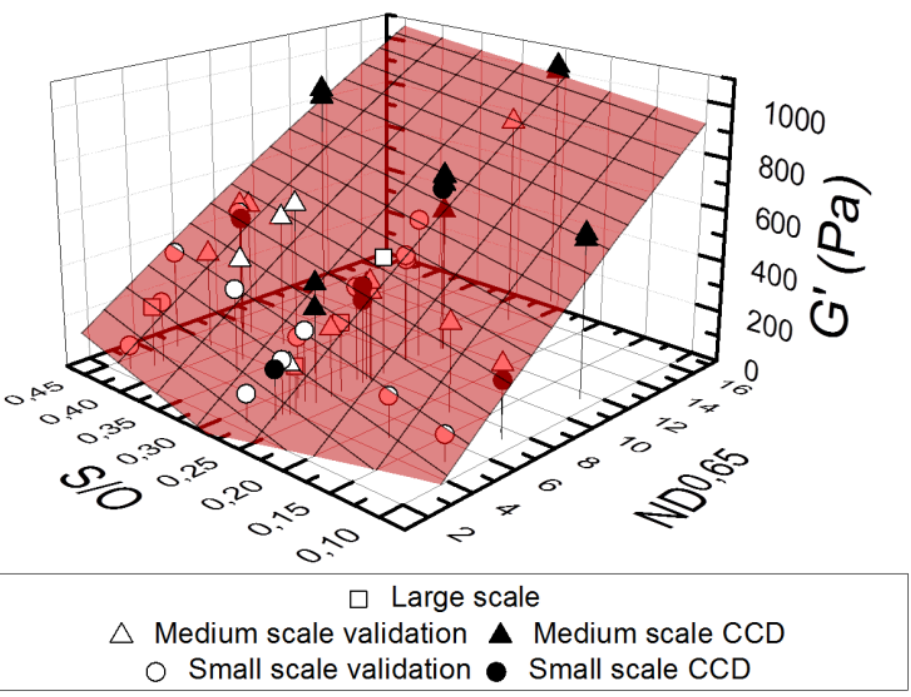

Fig. 18. Scale-up general model $(\alpha=0.65)$ and all the experimental values (CCD and validation $)(t=5.08 \mathrm{~min})$.
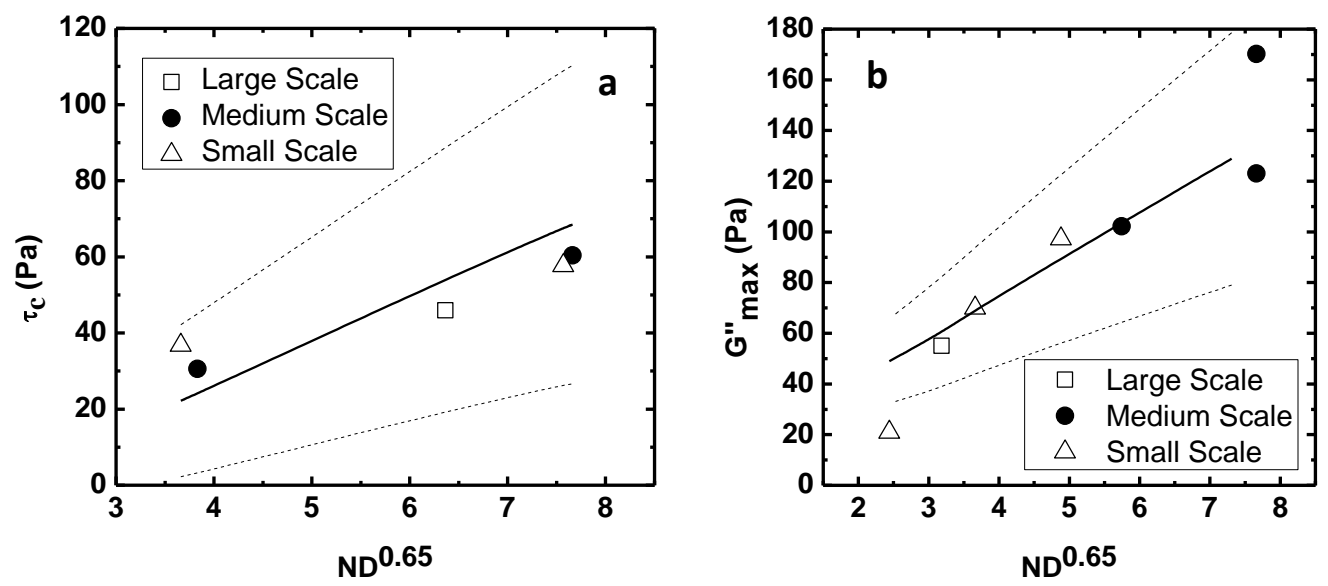

Fig. 19. (a) Scale-up general model for $\tau_{c}(\alpha=0.65)$ and the validation experimental points $\left(t=18.24\right.$ min and $S / O=0.43 \pm 0.02$ ). (b) Scale-up general model for $G{ }^{\prime \prime}{ }_{\text {max }}(\alpha=0.65)$ and the validation experimental points $(t=5.08 \mathrm{~min}$ and $S / O=0.43 \pm 0.02)$.

460 If we take the value of $\alpha=0.65$ and apply it to the emulsion change in BS after 30 min (Fig. 20-

461 a) and 24 hours (Fig. 20-b) of preparation, we can observe that, despite the dispersion in the 462 values at 24 hours, there is not an appreciable difference between the stability comparing the 463 three scales. The values agree with the model at $N D^{0.65}$, so the emulsions formed are similar at 464 all three scales and have the same stability. Also, the emulsions prepared at a higher stirring rate are more stable, as observed in [7]. 

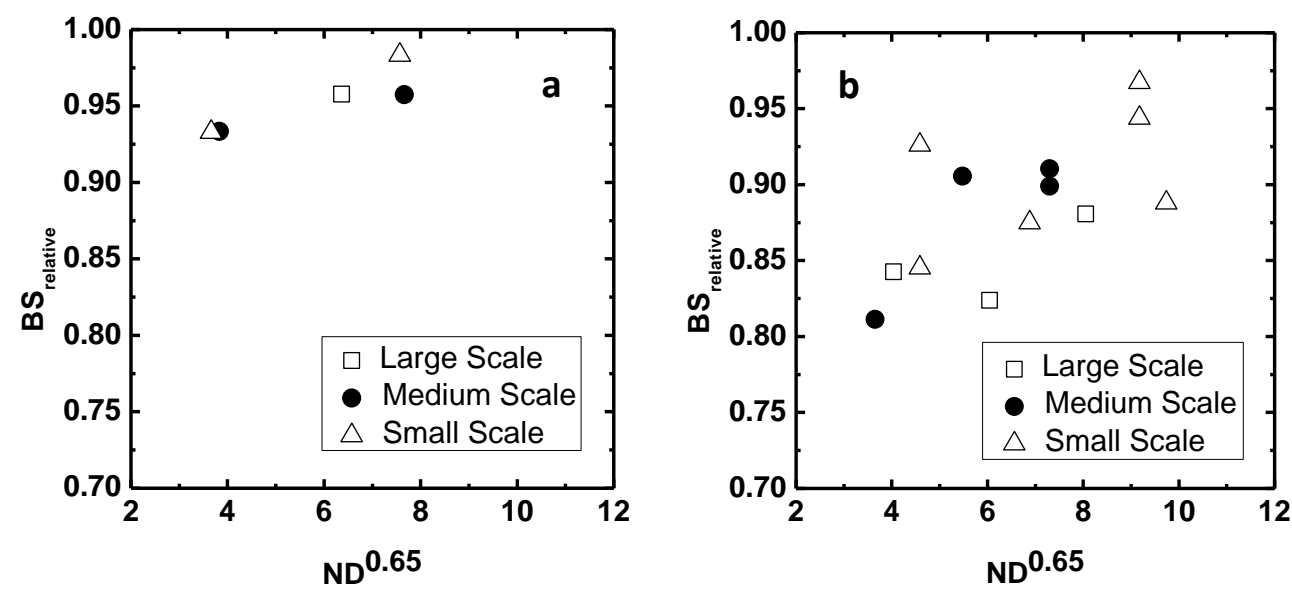

466

467

468

469

470

471

472

473

Fig. 20. (a) Relative backscattering $\left(\mathrm{BS}_{\mathrm{rel}}\right) 30 \mathrm{~min}$ after the emulsion preparation at $S / O=0.43$ and $t=18.24$ min. (b) Relative backscattering $\left(\mathrm{BS}_{\mathrm{rel}}\right) 24$ hours after the emulsion preparation at $S / O=0.267$ and $t=5.08 \mathrm{~min}$.

\subsection{Physical meaning of the power law exponent $\alpha$}

The power law exponent for this system is $\alpha=0.65$, which can be approximated to $\alpha=2 / 3$.

According to the table presented in [7], where the physical meaning of the power law exponents is described, that indicates the scale invariant of this process is the power per unit volume $P / V$ : emulsions with equal $P / V$ value should have the same properties. The expression for this parameter (2) is obtained from the definition of the power number $\left(\mathrm{N}_{\mathrm{p}}=P /\left(\rho \cdot N^{3} \cdot D^{5}\right)\right)$, where $\rho$ is the fluid density, and the volume of the vessel $\left(V=\pi \cdot\left(B^{2} / 4\right) \cdot H\right)$.

$P / V=\mathrm{N}_{\mathrm{p}} \cdot \rho \cdot N^{3} \cdot D^{5} /\left((\pi / 4) \cdot B^{2} \cdot H\right)$

The value of the exponent $\alpha=2 / 3$ is not intuitive but is derived from the equation when considering geometric similarity between vessels and their shape $\left(B_{1} / B_{2}=H_{1} / H_{2}=D_{1} / D_{2}\right)$. Using this relation, for a stirring rate at small scale equal to $1000 \mathrm{rpm}$, the stirring rate at medium scale would be $630 \mathrm{rpm}$ and at large scale $397 \mathrm{rpm}$. At large scale, the blades of the impeller had to be shortened $\left(D_{3}=16 \mathrm{~cm}\right)$ from what they should measure for keeping geometric similarity $(18 \mathrm{~cm})$. This change could introduce some errors to the models and conclusions derived from them, especially when extrapolating these findings to other emulsification systems. Nevertheless, the model derived from this system and the scale-up 
criterion encountered yield fair good predictions for the experimental data at the three different scales used in this work.

This scale-up criterion is well-known and has been analyzed in previous studies. Okufi et al [24] studied $P / V$ as a scale invariant at liquid - liquid dispersions, but they concluded that the equal tip speed was a better scale-up criterion. Bourne and $\mathrm{Yu}[25]$ assumed $P / V$ as a scale invariant in their study of macromixing at stirred tanks and several authors (Wilkens et al. [26], Chen et al. [27]) recommend $P / V$ to perform the scale-up of mixing processes. However, it is the first time, as far as we know, that it is deduced indirectly, without needing to measure neither the power nor the changing volume of the emulsion.

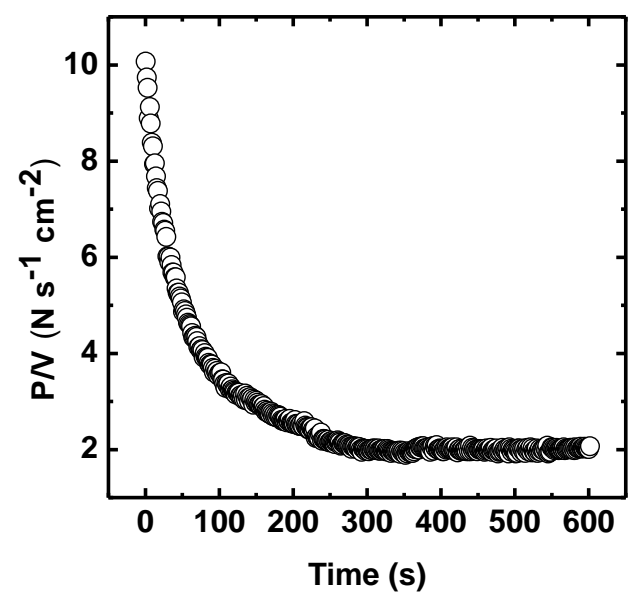

Fig. 21. Evolution of $P / V$ at $S / O=0.267, N=700 \mathrm{rpm}$ and $t=5.08 \mathrm{~min}$ at medium scale over time

In this study emulsions are formed by continuously adding the dispersed phase to the continuous phase, so the emulsion volume $V$ increases over time until all the dispersed phase is added. On the other hand, torque $T$ profiles obtained during the process show that this value increases while the dispersed phase is being added, since the more fluid volume, and the more viscous it is, the more energy input the stirrer has to provide to maintain the fixed stirring rate. $P / V$ is not constant over time, since the increase in volume is much more important than the increase in torque (Fig. 21). Hence, the interpretation of the power law exponent physical meaning is not obvious, since $P / V$ cannot be calculated from the beginning neither it cannot be

504 fixed as a scale invariant before experimentation. 
In the first part of this study, highly concentrated w/o emulsions were prepared following central composite designs at two different scales. We identified the factors that significantly affect the rheological parameters of the emulsions. We then constructed empirical models, from which we represented the response surfaces to visualize the variation of each rheological parameter with the process variables. In addition, we validated the models by performing additional experiments following simple designs. Although most of the rheological parameters followed the same behavior as the model predictions, since the models were obtained from a

513 limited number of experiments, they showed limited ability in predicting output parameters, 514 even within the range of factors studied.

515 Our main goal was to identify the process conditions that have to be kept constant at several scales to obtain highly concentrated emulsions with similar properties We conclude, first, that a methodology of scaling up can be deduced, based on maximizing the regression coefficient of different experimental data sets to obtain the missing parameters.

Secondly, in vessels of different size with geometric similarity, once the dispersed phase volume fraction, the surfactant concentration and the addition time of dispersed phase are fixed, the unknown variable is the stirring rate at each scale. The stirring rate has the greatest influence on the emulsion features, and it also depends on the production scale. For the preparation of highly concentrated w/o emulsions, the scale invariant related to the stirring rate and scale has the form $N D^{\alpha}$. An average value of $\alpha$ close to $2 / 3$ was found, when characterizing the emulsions by their rheological parameters. This result implies that the scale invariant for systems of this kind is equal to maintaining the power input per unit volume $(P / V)$ constant, which is a common scale-up criterion in mixing processes. 
531 The authors are grateful to the Spanish Ministry of Economy and Competitiveness for

532 supporting this work in the framework of the project CTQ2011-29336-C03-02.

Appendix A. Supporting information

\section{References}

[1] J.-L. Blin, R. Bleta, J. Ghanbaja, M.-J. Stébé, Fluorinated emulsions: Templates for the direct preparation of macroporous-mesoporous silica with a highly ordered array of large mesopores, Microporous Mesoporous Mater. 94 (2006) 74-80.

H. Zhang, A.I. Cooper, Synthesis and applications of emulsion-templated porous

[3] B.P. Binks, Macroporous silica from solid-stabilized emulsion templates, Adv. Mater. 14 (2002) 1824-1827.

[4] N. Du, M.-J. Stébé, R. Bleta, J.-L. Blin, Preparation and characterization of porous silica
templated by a nonionic fluorinated systems, Colloids Surfaces A Physicochem. Eng. Asp. 357 (2010) 116-127.

[5] A. Menner, K. Haibach, R. Powell, A. Bismarck, Tough reinforced open porous polymer foams via concentrated emulsion templating, Polymer (Guildf). 47 (2006) 7628-7635.

[6] A. Imhof, D.J. Pine, Ordered macroporous materials by emulsion templating, Nature. 389 (1997) 948-950.

[7] A. May-Masnou, M. Porras, A. Maestro, C. González, J.M. Gutiérrez, Scale invariants in the preparation of reverse high internal phase ratio emulsions, Chem. Eng. Sci. 101 (2013) 721-730.

[8] C.F. Welch, G.D. Rose, D. Malotky, S.T. Eckersley, Rheology of high internal phase emulsions, Langmuir. 22 (2006) 1544-50.

[9] R. Pal, Rheology of high internal phase ratio emulsions, Food Hydrocoll. 20 (2006) 9971005.

[10] N. Jager-Lézer, J.-F. Tranchant, V. Alard, C. Vu, P.C. Tchoreloff, J.-L. Grossiord, Rheological analysis of highly concentrated w/o emulsions, Rheol. Acta. 37 (1998) 129138. 
[11] I. Masalova, R. Foudazi, A.Y. Malkin, The rheology of highly concentrated emulsions stabilized with different surfactants, Colloids Surfaces A Physicochem. Eng. Asp. 375 (2011) 76-86.

[12] A. May-Masnou, K. Aramaki, J.M. Gutiérrez, Phase behavior and rheological analysis of reverse Llquid crystals and W/I2 and W/H2 gel emulsions using an amphiphilic block copolymer, Langmuir. 27 (2011) 2286-2298.

[13] M.M. Alam, Y. Sugiyama, K. Watanabe, K. Aramaki, Phase behavior and rheology of oil-swollen micellar cubic phase and gel emulsions in nonionic surfactant systems, J. Colloid Interface Sci. 341 (2010) 267-72.

[14] S.R. Derkach, Rheology of emulsions, Adv. Colloid Interface Sci. 151 (2009) 1-23.

[15] R. Pons, P. Erra, C. Solans, J.C. Ravey, M.-J. Stébé, Viscoelastic properties of gelemulsions: their relationship with structure and equilibrium properties, J. Phys. Chem. 97 (1993) 12320-12324.

[16] Y. Matsumoto, M.M. Alam, K. Aramaki, Phase behavior, formation, and rheology of cubic and hexagonal phase based gel emulsions in water/tetraglyceryl lauryl ether/oil systems, Colloids Surfaces A Physicochem. Eng. Asp. 341 (2009) 27-32.

[17] H. Kunieda, Y. Fukui, H. Uchiyama, C. Solans, Spontaneous formation of highly concentrated water-in-oil emulsions (gel-emulsions), Langmuir. 12 (1996) 2136-2140.

[18] K. Hyun, S.H. Kim, K.H. Ahn, S.J. Lee, Large amplitude oscillatory shear as a way to classify the complex fluids, J. Nonnewton. Fluid Mech. 107 (2002) 51-65.

[19] K. Hyun, J.G. Nam, M. Wilhelm, K.H. Ahn, S.J. Lee, Nonlinear response of complex fluids under LAOS (large amplitude oscillatory shear) flow, Korea-Australia Rheol. J. 15 (2003) 97.

[20] J. Aubin, C. Xuereb, Design of multiple impeller stirred tanks for the mixing of highly viscous fluids using CFD, Chem. Eng. Sci. 61 (2006) 2913-2920.

[21] T. Kumaresan, J.B. Joshi, Effect of impeller design on the flow pattern and mixing in stirred tanks, Chem. Eng. J. 115 (2006) 173-193.

[22] R. Pal, Yield stress and viscoelastic properties of high internal phase ratio emulsions, Colloid Polym. Sci. 277 (1999) 583-588.

[23] M. Capdevila, A. Maestro, M. Porras, J.M. Gutiérrez, Preparation of Span 80/oil/water highly concentrated emulsions: influence of composition and formation variables and scale-up, J. Colloid Interface Sci. 345 (2010) 27-33.

[24] S. Okufi, D. Ortiz, E.S. Perez, H. Sawistowski, Scale-up of liquid-liquid dispersions in stirred tanks, Can. J. Chem. Eng. 68 (1990) 400-406.

[25] J.R. Bourne, S. Yu, Investigation of micromixing in stirred tank reactors using parallel reactions, Ind. Eng. Chem. Res. 33 (1994) 41-55.

[26] R.J. Wilkens, C. Henry, L.E. Gates, How to scale-up mixing processes in nonNewtonian fluids, Chem. Eng. Prog. 99 (2003) 44-52. 
599

600

601

602

603

[27] J.P. Chen, F.B. Higgins, S.-Y. Chang, Y.-T. Hung, Mixing, in: L.K. Wang, Y.-T. Hung, N.K. Shammas (Eds.), Handb. Environ. Eng. Vol. 3 Physicochem. Treat. Process., 2005: pp. 47-101.

Published in: Chemical Engineering Science, Volume 111, 24 May 2014, Pages 410-420 